\section{A Further Hazard of Intravenous Therapy?}

Modern technology coupled with a little manual dexterity permits ready access to human blood vessels so that drugs and fluids can be administered and pressures recorded. While there is little doubt that these techniques have helped to reduce mortality in critically ill patients many types of iatrogenic disease have resulted: volume overload, ${ }^{1}$ air embolism, ${ }^{2}$ local damage to vessels with resulting thrombosis, ${ }^{3}$ and sepsis ${ }^{4}$ are well-recognized complications.

Traditionally, blood and other fluids for intravenous use have been administered from glass bottles, but they are difficult to store, they crack, and the rubber bungs may act as a nidus for infection. ${ }^{5}$ When bungs and bottles are reused particles of rubber may become dislodged and form emboli. These difficulties-and the shortage of glass containers-have led to more widespread use of pliable storage bags made of polyvinylchloride (PVC) plasticized with esters of benzene dicarboxylic acid (phthalic acid). The most widely used plasticizer is di-2-ethylhexyl phthalate (DEHP), which may constitute up to $40 \%$ of the dry weight of vinyl plastics in current medical use. ${ }^{6}$ Early laboratory studies indicated that DEHP had an extremely low toxicity, ${ }^{7}$ but this conclusion was questioned when it was shown that plastics used in medical practice had a cardiodepressant effect. ${ }^{8}$ Further doubts were cast on the safety of DEHF when it was shown to have accumulated in the tissues of two patients who had received blood transfusions during their terminal illness. ${ }^{9}$ Later it was found that DEHF given intravenously accumulates in the lungs ${ }^{6}$ and that urinary elimination is slow. In addition DEHP is partially metabolized to phthalic acid and an alcohol, 2-ethyl hexanol, ${ }^{610}$ both of which are more toxic than the parent compound.

Though at first sight these findings are alarming, there are only a few clinical occasions in which quantities of DEHF of any practical importance could enter the systemic circulation. These include transfusion with blood which has been stored for 48 hours or longer, ${ }^{11}$ extracorporeal circulation during cardiopulmonary bypass or haemodialysis, ${ }^{12}$ and umbilical catheterization in neonates. ${ }^{13}$ Recently Hillman and her colleagues have found DEHP in cardiac muscle and gut residues of infants dying of necrotizing enterocolitis-a condition which predominantly affects small, premature babies but may also occur in full-term infants after exchange transfusion. The latter association has led to suggestions ${ }^{13}$ that DEHP might produce intravascular coagulation. Though this hypothesis is plausible it seems more likely that intravascular coagulation follows sepsis or excessive intravascular pressure during the exchange procedure. It is reassuring that recent studies ${ }^{14}$ have failed to show that phthalates have any effect on platelet function; furthermore, the cardiodepressant effectlof substances leached from older types of PVC tubing was probably related to the stabilizers rather than to DEHP. ${ }^{15}$ Nonetheless, it is clear that further investigations of potential vascular toxic effects of DEHP and other plasticizers will be needed before they can be regarded as totally safe. Until such information is available the use of PVC containers and cannulae should be restricted to well-defined clinical indications.

1 Downs, J. W., Annals of Surgery, 1958, 148, 73.

2 Mollinson, P. L., in Blood Transfusions in Clinical Medicine, 5th edn. p. 586. Oxford, Blackwell, 1972.

3 McNair, T. J., and Dudley, H. A. F., Lancet, 1959, 2, 365.

4 British Medical fournal, 1972, 3, 190.
${ }^{5}$ Garvan, J. M., and Gunner, B. W., Medical Fournal of Australia, 1963, 2,140

${ }^{6}$ Daniel, J. W., and Bratt, H., Toxicolozy, 1974, 2, 51.

7 Shaffer, C. B., Carpenter, C. P., and Smyth, H. E., Fournal of Industrial Hygiene and Toxicology, 1945, 27, 130.

${ }^{8}$ Meyers, D. B., Autian, J., and Guess, W. L., Fournal of Pharmaceutical Sciences, 1964, 53, 774.

${ }^{9}$ Jaeger, R. J., and Rubin, R. J., Science, 1970, 170, 460.

10 Carter, J. E., Roll, D. B., and Petersen, R. V., Drug Metabolism and Disposition, 1974, 2, 341 .

11 Jaeger, R. J., and Rubin, R. J., Lancet, 1970, 2, 151

12 Jaeger, R. J., and Rubin, R. J., New England fournal of Medicine, 1972 , 287, 1114 .

${ }^{13}$ Hillman, L. S., Goodwin, S. L., and Sherman, W. R., New England Fournal of Medicine, 1975, 292, 381.

14 Valeri, C. R., et al., Environmental Health Perspectives, 1973, 3, 103.

${ }^{15}$ Meyler, F. L., Willebrands, A. F., and Duner, D., Circulation Research, $1960,8,44$.

\section{Early Thymectomy for Myasthenia Gravis}

It has long been recognized in Britain that the earlier thymectomy was performed in myasthenia gravis the better the prognosis. ${ }^{2}$ There was, however, for many years a natural reluctance to submit patients whose symptoms were readily controlled with anticholinesterase drugs to an operation which carried a definite mortality. More recently the risks of thymectomy have been dramatically reduced as a result of advances in the management of ventilatory failure with assisted respiration and the better recognition of myasthenic and cholinergic crises, and as the numbers of patients undergoing operation have increased its benefits have become more apparent. The incidence of remission increases with the number of years after thymectomy. Perlo et al. ${ }^{2}$ in Boston and New York reported complete remission or substantial improvement in $84 \%$ of 45 women with severe myasthenia without a thymic tumour. Edward and Wilson ${ }^{3}$ found improvement in $80 \%$, and Papatestas and his colleagues ${ }^{4}$ found that five years after thymectomy $90 \%$ of their patients were in remission or had shown considerable improvement.

The indications for thymectomy are, therefore, expanding to include milder cases and patients with earlier disease. In a recent review ${ }^{5}$ of progress in myasthenia the indications for surgery at the various major centres concerned with the care of myasthenic patients was quoted. At the Mount Sinai Hospital, New York, with experience of 185 patients with myasthenia, the indications for surgery in patients without a tumour were an increasing need for anticholinesterase drugs or a poor response to medication. ${ }^{4}$ The indications prompting thymectomy in Liverpool were severe weakness and incapacity despite anticholinesterase therapy, recurring respiratory infections leading to one or more incidents of myasthenic or cholinergic crises, and recently the operation has been advised for married women with hopes of having children. ${ }^{3}$ At the New End Endocrine Clinic (now at the New Royal Free Hospital) over 260 patients with myasthenia gravis have been treated and thymectomy was offered to most of these, with the exception of patients with the purely ocular form of the disease..$^{5}$

A new study from Mount Sinai Hospital ${ }^{6}$ has emphasized the value of early surgery. Genkins et al. have experience of 353 patients who have undergone thymectomy, and their evidence shows that the duration of disease and the presence of germinal centres within the thymus gland influence the response to operation. The shortest interval between thym- 